\title{
Positive Solutions of a Diffusive Predator-Prey System including Disease for Prey and Equipped with Dirichlet Boundary Condition
}

\author{
Xiaoqing Wen, ${ }^{1}$ Yue Chen, ${ }^{2}$ and Hongwei Yin ${ }^{1,3}$ \\ ${ }^{1}$ School of Sciences, Nanchang University, Nanchang 330031, China \\ ${ }^{2}$ School of Civil Engineering and Architecture, Nanchang University, Nanchang 330031, China \\ ${ }^{3}$ Numerical Simulation and High-Performance Computing Laboratory, School of Sciences, Nanchang University, \\ Nanchang 330031, China
}

Correspondence should be addressed to Hongwei Yin; hongwei-yin@hotmail.com

Received 30 June 2016; Accepted 14 September 2016

Academic Editor: Sanja Konjik

Copyright (C) 2016 Xiaoqing Wen et al. This is an open access article distributed under the Creative Commons Attribution License, which permits unrestricted use, distribution, and reproduction in any medium, provided the original work is properly cited.

We study a three-dimensional system of a diffusive predator-prey model including disease spread for prey and with Dirichlet boundary condition and Michaelis-Menten functional response. By semigroup method, we are able to achieve existence of a global solution of this system. Extinction of this system is established by spectral method. By using bifurcation theory and fixed point index theory, we obtain existence and nonexistence of inhomogeneous positive solutions of this system in steady state.

\section{Introduction}

In recent decade, predator-prey systems are significant in mathematical biology and applications [1-3]. These systems are usually described by ordinary differential equation (ODEs). Besides, epidemic models have been deeply studied by differential equations in $[4,5]$, based on the pioneer work of the classical SIR model by Kermack and Mckendrick. However, epidemic disease can spread within a species except for interactions among species. For this case, some ecoepidemiological models were introduced in [6-8] to study how disease affects the dynamic of predator-prey systems. Since we live in the real world, spatial factor plays an important role in populations dynamic. Moreover, space could induce some significant phenomena different from the corresponding ODE systems. At present, a lot of literature has showed that predator-prey systems with spatial diffusion can produce a variety of pattern formation by Turing bifurcation [9-11] and references wherein. In this paper, we are interested in the following model having Holling-type II functional response with disease in the prey:

$$
\begin{aligned}
u_{t}-\Delta u & =u(a-u-v)-\beta u v, \\
v_{t}-\Delta v & =\beta u v-b v-\frac{c w v}{m w+v}, \quad(x, t) \in \Omega \times \mathbb{R}^{+}, \\
w_{t}-\Delta w & =-d w+\frac{e w v}{m w+v}, \quad(x, t) \in \Omega \times \mathbb{R}^{+},
\end{aligned}
$$

There are two species in the model: prey $(u+v)$ and predator $(w)$. In (1a), $u$ is susceptible prey, and $v$ denotes infected prey. Only susceptible prey $u$ is capable of reproducing and its reproducing rate is $a$. However, the infected prey does possess the capability of reproducing and still contributes to population growth of susceptible prey $u$ according to the logistic growth law. The disease is transmitted only within prey in the form of $\beta u v$, and $\beta \in(0,1)$ is called the transmission coefficient. The disease is not genetically inherited. The infected populations do not recover or become immune. Infected 
individuals are less active and can be caught more easily by predator resulting in increasing survival of susceptible prey. Catching rate of predators is a ratio-dependent MichaelisMenten functional response function; that is, $c w v /(m w+v)$. Infected prey has a death rate $b>0$ and $d>0$ is a death rate of predators. Prey and predators are inhabited in a bounded region $\Omega \subseteq \mathbb{R}^{N}$ with the smooth boundary $\partial \Omega$, where $N$ is a spatial dimension number. $\Delta$ is the Laplace operator and represents diffusion of individuals from a high density region to low density one. Zhang et al. [12] have studied (1a) under the homogeneous Neumann boundary condition and obtain permanence and stability.

However, few author, as far as we know, investigated mathematical property of (1a) equipped with Dirichlet boundary condition, such as existence of global timedependent positive solution and inhomogeneous positive solutions in steady state. To this end, in this paper we make the first attempt to fill this gap and study existence of a global positive solution (1a) with homogeneous Dirichlet boundary conditions:

$$
u(x, t)=v(x, t)=w(x, t)=0, \quad(x, t) \in \partial \Omega \times \mathbb{R}^{+},
$$

which imply that predator and prey die out on the boundary $\partial \Omega$ and initial values

$$
\begin{gathered}
u(x, 0)=u_{0}(x) \geq 0, \\
v(x, 0)=v_{0}(x) \geq 0, \\
w(x, 0)=w_{0}(x) \geq 0,
\end{gathered}
$$

$x \in \Omega$.

Besides, we first attempt to investigate existence and nonexistence of inhomogeneous positive solutions of (1a), (1b), and (1c) in steady state as follows:

$$
\begin{aligned}
-\Delta u & =u(a-u-v)-\beta u v, \quad x \in \Omega, \\
-\Delta v & =\beta u v-b v-\frac{c w v}{m w+v}, \quad x \in \Omega, \\
-\Delta w & =-d w+\frac{e w v}{m w+v}, \quad x \in \Omega, \\
u & =v=w=0, \quad x \in \partial \Omega .
\end{aligned}
$$

The remainder of the paper is organized as follows: in Section 2, some necessary notations and theoretical results are introduced; in Section 3, we examine existence of a global positive solution to (1a), (1b), and (1c); in Section 4 we discuss extinction of system (1a), (1b), and (1c); existence and nonexistence of inhomogeneous positive solutions are discussed by bifurcation theory and degree theory in Section 5.

\section{Preliminary}

In this section, some notations and basic well-known results are introduced. For any $q \in C(\bar{\Omega})$, the linear eigenvalue problem $[13,14]$,

$$
\begin{array}{r}
-\Delta u+q(x) u=\lambda u, \quad x \in \Omega, \\
u=0, \quad x \in \partial \Omega,
\end{array}
$$

has an infinite sequence of eigenvalues. Let $\lambda_{1}(q)$ be the principal eigenvalue of (9) which is simple and its eigenfunction does not change sign in $\Omega$. We denote $\lambda_{1}(0)$ by $\lambda_{1}$ for simplicity and the eigenfunction corresponding to $\lambda_{1}$ is denoted by $\varphi_{1}(x) \cdot q_{1}(x) \leq q_{2}(x)$ with $q_{1}$ being not identically equal to $q_{2}$ implies $\lambda_{1}\left(q_{1}\right) \leq \lambda_{1}\left(q_{2}\right)$.

Now, consider the nonlinear boundary value problem

$$
\begin{aligned}
-\Delta u & =u(a-u), \quad x \in \Omega, \\
u & =0, \quad x \in \partial \Omega .
\end{aligned}
$$

If $a>\lambda_{1}$ then (4) has a unique positive solution (cf. $[15,16]$ ). We denote this unique positive solution by $u_{a}$ and $u_{a}<a$.

Next, the fixed point index theory, which is used later, is introduced. Let $E$ be a real Banach space. $W$ is called a wedge if $W$ is a closed convex set and $\beta W \subset W$ for all $\beta \geq 0$. For $y \in W$, we define

$$
\begin{aligned}
& W_{y}=\{x \in E: \exists r=r(x)>0, \text { s.t. } y+r x \in W\}, \\
& S_{y}=\left\{x \in \bar{W}_{y}:-x \in \bar{W}_{y}\right\} .
\end{aligned}
$$

We always assume that $E=\overline{W-W}$. Let $T: W_{y} \rightarrow W_{y}$ be a compact linear operator on $E$. We say that $T$ has property $\alpha$ on $\bar{W}_{y}$ if there exists $t \in(0,1)$ and $w \in \bar{W}_{y} \backslash S_{y}$ such that $(1-t T) w \in S_{y}$. Let $A: W \rightarrow W$ be a compact operator with a fixed point $y \in W$ and $A$ is Fréchet differentiable at $y$. Let $L=A^{\prime}(y)$ be the Fréchet derivative of $A$ at $y$. Then $L$ maps $\bar{W}_{y}$ into itself. We denote by $\operatorname{index}_{W}(A, y)$ the fixed point index of $A$ at $y$ relative to $W$.

Proposition 1 (see [16-18]). Assume that $I-L$ is invertible on $\bar{W}_{y}$. Then

(i) if L has property $\alpha$ on $\bar{W}_{y}$, then $\operatorname{index}_{W}(A, y)=0$;

(ii) if $L$ does not have property $\alpha$ on $\bar{W}_{y}$, then $\operatorname{index}_{W}(A$, $y)=(-1)^{\sigma}$, where $\sigma$ is the sum of multiplicities of all eigenvalues of $L$ which is greater than 1 .

For a linear operator $L$, we denote by $r(L)$ the spectral radius of $L$.

Proposition 2 (see $[16,18]$ ). Let $q(x) \in C(\bar{\Omega})$ and $M$ be a positive constant such that $M-q(x)>0$ on $\bar{\Omega}$. Let $\lambda_{1}(q)$ be the first eigenvalue of the problem

$$
\begin{aligned}
-\Delta u+q(x) u & =\lambda u, \quad x \in \Omega, \\
u & =0, \quad x \in \partial \Omega .
\end{aligned}
$$

We have the following conclusions:
(a) $\lambda_{1}(q)<0 \Rightarrow r\left[(M-\Delta)^{-1}(M-q(x))\right]>1$,
(b) $\lambda_{1}(q)>0 \Rightarrow r\left[(M-\Delta)^{-1}(M-q(x))\right]<1$,
(c) $\lambda_{1}(q)=0 \Rightarrow r\left[(M-\Delta)^{-1}(M-q(x))\right]=1$.

Now, we introduce the degree calculations, which was introduced by Dancer and Du in [19]. Assume that $E_{1}$ and $E_{2}$ 
are ordered Banach spaces with positive cones $W_{1}$ and $W_{2}$, respectively. Let $E=E_{1} \oplus E_{2}$ and $W=W_{1} \oplus W_{2}$. Then $E$ is an ordered Banach space with positive cone $W$. Let $D$ be an open set in $W$ containing 0 and $F_{i}: \bar{D} \rightarrow W_{i}$ be completely continuous operators, $i=1,2$. Denote by $(u, v)$ a general element in $W$ with $u \in W_{1}$ and $v \in W_{2}$. Let $F: \bar{D} \rightarrow W$ be defined by

$$
F(u, v)=\left(F_{1}(u, v), F_{2}(u, v)\right) .
$$

Define $W_{2}(\epsilon)=\left\{v \in W_{2}:\|v\|_{E_{2}}<\epsilon\right\}$.

Theorem 3 (see [15]). Suppose that $U \subset W_{1} \cap D$ is relatively open and bounded and

$$
\begin{array}{ll}
F_{1}(u, 0) \neq u & \text { for } u \in \partial U \\
F_{2}(u, 0)=0 & \text { for } u \in \bar{U}
\end{array}
$$

Suppose that $F_{2}: D \rightarrow W_{2}$ extends to a continuously differentiable mapping of a neighborhood of $D$ into $E_{2}, W_{2}-W_{2}$ is dense in $E_{2}$, and $\Phi=\left\{u \in U: u=F_{1}(u, 0)\right\}$. Then the following are true:

(i) $\operatorname{deg}_{W}\left(I-F, U \times W_{2}(\varepsilon), 0\right)=0$ for $\varepsilon>0$ small, if, for any $u \in \Phi$, the spectral radius $r\left(\left.F_{2}^{\prime}(u, 0)\right|_{W_{2}}\right)>1$ and 1 is not an eigenvalue of $\left.F_{2}^{\prime}(u, 0)\right|_{W_{2}}$ corresponding to a positive eigenvector;

(ii) $\operatorname{deg}_{W}\left(I-F, U \times W_{2}(\varepsilon), 0\right)=\operatorname{deg}_{W}\left(I-\left.F_{1}\right|_{W_{1}}, U, 0\right)$ for $\varepsilon>0$ small, if, for any $u \in \Phi$, the spectral radius $r\left(\left.F_{2}^{\prime}(u, 0)\right|_{W_{2}}\right)<1$.

\section{Existence of a Global Positive Solution of (1a), (1b), and (1c)}

For convenience, we introduce some notations. For $x, y \in \mathbb{R}$, we denote $x \wedge y:=\min \{x, y\}, x \vee y:=\max \{x, y\}, x_{+}:=x \vee 0$, and $x_{-}:=(-x) \vee 0$ and extend these notations to real-valued functions. If $(V, \geq)$ is partially ordered vector space, we denote its positive cone by $V_{+}:=\{v \in V: v \geq 0\}$. For $p \in[1,+\infty)$, $p>N / 2$, let $X=\left\{L^{p}(\Omega)\right\}^{3}$. Assume $z=(u, v, w) \in X$ and its norm is defined as $\|z\|=\|u\|_{p}+\|v\|_{p}+\|w\|_{p}$.

Hence, system (1a), (1b), and (1c) can be rewritten as an abstract differential equation:

$$
\begin{aligned}
\frac{d z}{d t} & =A z+H(z), \quad t \in \mathbb{R}^{+}, \\
z(0) & =z_{0}, \quad t=0,
\end{aligned}
$$

where $z=(u(x, t), v(x, t), w(x, t))^{T}$ and $z_{0}=\left(u_{0}, v_{0}, w_{0}\right)^{T}$,

$$
A=\left(\begin{array}{lll}
\Delta & 0 & 0 \\
0 & \Delta & 0 \\
0 & 0 & \Delta
\end{array}\right)
$$

with

$$
\begin{aligned}
D(A)= & \left\{(u, v, w) \in\left\{C^{2}(\bar{\Omega})\right\}^{3},\left.u\right|_{\partial \Omega}=\left.v\right|_{\partial \Omega}=\left.w\right|_{\partial \Omega}\right. \\
= & 0\}, \\
H(z)= & \left(\begin{array}{c}
u(a-u-v)-\beta u v \\
\beta u v-b v-\frac{c w v}{m w+v} \\
-d w+\frac{e w v}{m w+v}
\end{array}\right) .
\end{aligned}
$$

Next, the existence of the local solution for (9) will be proved. First, we give out the following lemma.

Lemma 4. For every $z_{0} \in X_{+}$, Cauchy problem (9) has a unique maximal local solution

$$
\begin{gathered}
z \in C\left(\left[0, T_{\max }\right) ; X\right) \cap C^{1}\left(\left(0, T_{\max }\right) ; X\right) \\
\cap C\left(\left(0, T_{\max }\right) ; D\left(A_{p}\right)\right),
\end{gathered}
$$

which satisfies the following Duhamel formula for $t \in\left[0, T_{\max }\right)$ :

$$
z(t)=e^{t A_{p}} z_{0}+\int_{0}^{t} e^{(t-s) A_{p}} H[z(s)] d s
$$

where $A_{p}$ is a operator and the closure of $A$ in $X$. Moreover, if $T_{\max }<\infty$ then $\lim \sup _{T_{\max }^{-}}\|z(t)\|=\infty$.

Proof. Since the operator $A_{p}$ is the closure of $A$ in $X$, it generates an analytic, condensed, strong continuous operator semigroup $e^{t A_{p}}$. Moreover, we observe that $H: D\left(A_{p}\right) \rightarrow X$ is locally Lipschitz on a bounded set [20]. Via Theorem 7.2.1 in [21], we complete this proof.

Lemma 5. For initial-boundary problem (1a), (1b), and (1c), the components of its solution $u(x, t), v(x, t), w(x, t)$ are nonnegative.

Proof. To prove $u(x, t), v(x, t), w(x, t) \geq 0$ for $t \in\left[0, T_{\max }\right)$ and $x \in \Omega$, we consider the following system:

$$
\begin{aligned}
& \frac{\partial u^{\prime}}{\partial t}=\Delta u^{\prime}+u_{+}^{\prime}\left(a-u_{+}^{\prime}-v_{+}^{\prime}\right)-\beta u_{+}^{\prime} v_{+}^{\prime}, \\
& \frac{\partial v^{\prime}}{\partial t}=\Delta v^{\prime}+\beta u_{+}^{\prime} v_{+}^{\prime}-b v_{+}^{\prime}-\frac{c w_{+}^{\prime} v_{+}^{\prime}}{m w_{+}^{\prime}+v_{+}^{\prime}}, \\
& \frac{\partial w^{\prime}}{\partial t}=\Delta w^{\prime}-d w_{+}^{\prime}+\frac{e w_{+}^{\prime} v_{+}^{\prime}}{m w_{+}^{\prime}+v_{+}^{\prime}}
\end{aligned}
$$

with boundary and initial conditions

$$
\begin{aligned}
\left.u^{\prime}\right|_{\partial \Omega} & =\left.v^{\prime}\right|_{\partial \Omega}=\left.w^{\prime}\right|_{\partial \Omega}=0, \\
u^{\prime}(x, 0) & =u_{0}, \\
v^{\prime}(x, 0) & =v_{0}, \\
w^{\prime}(x, 0) & =w_{0} .
\end{aligned}
$$


After multiplying (14a), (14b), and (14c) by $u_{-}^{\prime}, v_{-}^{\prime}$, and $w_{-}^{\prime}$, respectively, and integrating by parts on the domain $\Omega$, we obtain

$$
\begin{aligned}
& -\frac{1}{2} \frac{d}{d t} \int_{\Omega}\left|u_{-}^{\prime}\right|^{2} d x-\int_{\Omega}\left|\nabla u_{-}^{\prime}\right|^{2} d x=0, \\
& -\frac{1}{2} \frac{d}{d t} \int_{\Omega}\left|v_{-}^{\prime}\right|^{2} d x-\int_{\Omega}\left|\nabla v_{-}^{\prime}\right|^{2} d x=0, \\
& -\frac{1}{2} \frac{d}{d t} \int_{\Omega}\left|w_{-}^{\prime}\right|^{2} d x-\int_{\Omega}\left|\nabla w_{-}^{\prime}\right|^{2} d x=0 .
\end{aligned}
$$

Hence, for $t \in\left(0, T_{\max }\right)$,

$$
\begin{aligned}
& \left\|u_{-}^{\prime}(\cdot, t)\right\|_{2}^{2}+\left\|v_{-}^{\prime}(\cdot, t)\right\|_{2}^{2} \leq\left\|u_{-}^{\prime}(\cdot, 0)\right\|_{2}^{2}+\left\|v_{-}^{\prime}(\cdot, 0)\right\|_{2}^{2} \\
& \quad=0 .
\end{aligned}
$$

Consequently,

$$
\begin{gathered}
u^{\prime}(x, t) \geq 0, \\
v^{\prime}(x, t) \geq 0, \\
w^{\prime}(x, t) \geq 0 .
\end{gathered}
$$

Now, we can know that $\left(u^{\prime}(x, t), v^{\prime}(x, t), w^{\prime}(x, t)\right)$ is a solution of (1a), (1b), and (1c), and according to Lemma 4, we finally get that

$$
\begin{aligned}
& u(x, t)=u^{\prime}(x, t) \geq 0, \\
& v(x, t)=v^{\prime}(x, t) \geq 0, \\
& w(x, t)=w^{\prime}(x, t),
\end{aligned}
$$

$$
t \in\left(0, T_{\max }\right)
$$

Next, we will prove the global existence of the positive solution for (1a), (1b), and (1c). Via Lemmas 4 and 5, we only need to show that the solution of (1a), (1b), and (1c) is uniformly bounded, that is, dissipation.

Theorem 6. Let $(u(x, t), v(x, t), w(x, t))$ be the solution of $(1 a)$, $(1 b)$, and (1c) with initial value (1c); then

$$
\begin{aligned}
& 0 \leq u(x, t) \leq c_{1}, \\
& 0 \leq v(x, t) \leq c_{2}, \\
& 0 \leq w(x, t) \leq c_{3}
\end{aligned}
$$

for $(x, t) \in \Omega \times\left[0, T_{\max }\right)$, where $c_{i}, i=1,2,3$ are defined by

$$
\begin{aligned}
& c_{1}:=\max \left\{a,\left\|u_{0}\right\|_{\infty}\right\}, \\
& c_{2}:=\max \left\{\frac{\beta(a+b) c_{1}}{b(1+\beta)}, \frac{\beta\left\|u_{0}\right\|_{\infty}}{1+\beta}+\left\|v_{0}\right\|_{\infty}\right\},
\end{aligned}
$$

$$
\begin{aligned}
c_{3} & :=\max \left\{\frac{(a+d) e \beta c_{1}}{c d(1+\beta)}\right. \\
& \left.+\frac{e c_{2}}{c}, \frac{e}{c}\left(\frac{\beta\left\|u_{0}\right\|_{\infty}}{1+\beta}+\left\|v_{0}\right\|_{\infty}\right)+\left\|w_{0}\right\|_{\infty}\right\} .
\end{aligned}
$$

Proof. In view of Lemma 5, we only prove the upper bound of (1a), (1b), and (1c) for $(x, t) \in \Omega \times\left[0, T_{\max }\right)$. From (1a), we have

$$
\begin{aligned}
u_{t} & \leq \Delta u+u(a-u), \quad \text { in } \Omega \times \mathbb{R}^{+}, \\
u(x, t) & =0, \quad \text { on } \partial \Omega \times \mathbb{R}^{+}, \\
u(x, 0) & =u_{0}, \quad \text { in } \Omega .
\end{aligned}
$$

We get $u(x, t) \leq c_{1},(x, t) \in \Omega \times\left(0, T_{\max }\right)$ by maximum principle, where $c_{1}=\max \left\{a,\left\|u_{0}\right\|_{\infty}\right\}$.

Let $v_{1}=\beta u /(1+\beta)+v$. From (1b) we have

$$
\begin{aligned}
\left(v_{1}\right)_{t}-\Delta v_{1} & =\frac{\beta}{1+\beta} u(a-u)-b v-\frac{c w v}{m w+v} \\
& \leq \frac{a \beta u}{1+\beta}-b\left(v_{1}-\frac{\beta u}{1+\beta}\right) \\
& \leq \frac{\beta(a+b) c_{1}}{1+\beta}-b v_{1} .
\end{aligned}
$$

Hence, by maximum principle we have that

$$
\begin{aligned}
v(x, t) & \leq v_{1}(x, t) \\
& \leq \max \left\{\frac{\beta(a+b) c_{1}}{b(1+\beta)}, \frac{\beta\left\|u_{0}\right\|_{\infty}}{1+\beta}+\left\|v_{0}\right\|_{\infty}\right\} \\
& :=c_{2}, \quad t \in[0, T) .
\end{aligned}
$$

By using the same method, we consider $w_{1}=(e / c)(\beta u /(1+$ $\beta)+v)+w$; then from (1c) we have that

$$
\begin{aligned}
\left(w_{1}\right)_{t}-\Delta w_{1}= & \frac{e \beta}{c(1+\beta)} u(a-u)-\frac{b e}{c} v-d w \\
\leq & \frac{a e \beta}{c(1+\beta)} u \\
& -d\left[w_{1}-\frac{e \beta u}{c(1+\beta)}-\frac{e v}{c}\right] \\
\leq & \frac{(a+d) e \beta c_{1}}{c(1+\beta)}+\frac{d e c_{2}}{c}-d w_{1} .
\end{aligned}
$$

Thus, the maximum principle shows that

$$
\begin{aligned}
& w(x, t) \leq w_{1}(x, t) \leq \max \left\{\frac{(a+d) e \beta c_{1}}{c d(1+\beta)}\right. \\
& \left.+\frac{e c_{2}}{c}, \frac{e}{c}\left(\frac{\beta\left\|u_{0}\right\|_{\infty}}{1+\beta}+\left\|v_{0}\right\|_{\infty}\right)+\left\|w_{0}\right\|_{\infty}\right\}:=c_{3} .
\end{aligned}
$$


Remark 7. From Theorem 6, we know that the upper bound of the solution of (1a), (1b), and (1c) is independent of the maximal time $T_{\max }$, which implies that the solution of (1a), (1b), and (1c) is a global solution.

By Lemmas 4 and 5 and Theorem 6, we can get the following theorem.

Theorem 8. System (1a), (1b), and (1c) has a unique, nonnegative, and bounded solution $z(x, t)=(u(x, t), v(x, t), w(x, t))$, such that

$$
\begin{gathered}
z(x, t) \in C([0, \infty), X) \cap C^{1}((0, \infty), X) \\
\cap C((x, \infty), D(A)) .
\end{gathered}
$$

\section{Extinction of (1a), (1b), and (1c)}

Theorem 9. Let $(u(x, t), v(x, t), w(x, t))$ be a positive solution of (1a), (1b), and (1c), then we have the following:

(i) if $a \leq \lambda_{1}$ and $e<d$ then $(u, v, w) \rightarrow(0,0,0)$ as $t \rightarrow$ $\infty$;

(ii) if $a<\lambda_{1}$ and $-b<\lambda_{1}\left(-\beta u_{a}\right)$ then $(u, v, w) \rightarrow$ $\left(u_{a}, 0,0\right)$ as $t \rightarrow \infty$.

Proof. (i) First, we observe that solution $(u, v, w)$ of (1a), (1b), and (1c) satisfies

$$
\begin{gathered}
u_{t}-\Delta u \leq u(a-u), \quad(x, t) \in \Omega \times \mathbb{R}^{+}, \\
u=0, \quad(x, t) \in \partial \Omega \times \mathbb{R}^{+} .
\end{gathered}
$$

Let $\varepsilon$ be a sufficiently small positive constant such that $\varepsilon \leq$ $b / \beta$. Since $a \leq \lambda_{1}$, we see that $u(x, t) \rightarrow 0$ uniformly as $t \rightarrow \infty$ by using comparison principle for elliptic problems. Then there exists $T_{\varepsilon} \geq 0$ such that $u(x, t) \leq \varepsilon$ for all $t \geq T_{\varepsilon}$. Therefore, we have

$$
\begin{gathered}
v_{t}-\Delta v \leq v(\beta \varepsilon-b), \quad(x, t) \in \Omega \times\left(T_{\varepsilon}, \infty\right), \\
v=0, \quad(x, t) \in \partial \Omega \times\left(T_{\varepsilon}, \infty\right),
\end{gathered}
$$

which concludes that $v(x, t) \rightarrow 0$ uniformly as $t \rightarrow \infty$. Similarly, there exists $T_{\varepsilon}^{\prime}>0$ such that $v(x, t) \leq \varepsilon$ for all $t>T_{\varepsilon}^{\prime}$. Further, since $e w v /(m w+v)$ is monotone increasing with respect to the variable $v$, we have

$$
\begin{gathered}
w_{t}-\Delta w \leq w\left(\frac{e \varepsilon}{m w+\varepsilon}-d\right) \leq w(e-d), \\
\quad(x, t) \in \Omega \times\left(T_{\varepsilon}^{\prime}, \infty\right), \\
w=0, \quad(x, t) \in \partial \Omega \times\left(T_{\varepsilon}^{\prime}, \infty\right),
\end{gathered}
$$

which concludes that $w(x, t) \rightarrow 0$ as $t \rightarrow \infty$.

(ii) Let $\varepsilon$ be a sufficiently small positive constant such that

$$
\begin{aligned}
& \text { (a) } \varepsilon<\frac{\lambda_{1}\left(b-\beta u_{a}\right)}{\beta}, \\
& \text { (b) } \varepsilon<\frac{a-\lambda_{1}}{\beta+1} .
\end{aligned}
$$

Since $a>\lambda_{1}$, we get from (28) and comparison theorem that

$$
\limsup _{t \rightarrow \infty} u(x, t) \leq u_{a} .
$$

Then, there exists $T_{\varepsilon}>0$ such that $u(x, t) \leq u_{a}+\varepsilon$ for all $t>T_{\varepsilon}$. Thus, we have

$$
\begin{aligned}
v_{t}-\Delta v & \leq v\left[\beta\left(u_{a}+\varepsilon\right)-b\right], \quad(x, t) \in \Omega \times\left(T_{\varepsilon}, \infty\right), \\
v & =0, \quad(x, t) \in \partial \Omega \times\left(T_{\varepsilon}, \infty\right) .
\end{aligned}
$$

Since $\varepsilon$ satisfies (a) and $-b<\lambda_{1}\left(-\beta u_{a}\right)$, we conclude that $v(x, t) \rightarrow 0$ uniformly as $t \rightarrow \infty$. Similarly, we can see that $w(x, t) \rightarrow 0$ uniformly as $t \rightarrow \infty$.

Besides, there exists $T_{\varepsilon}^{\prime} \geq 0$ such that $v(x, t), w(x, t)<\varepsilon$ for all $t>T_{\varepsilon}^{\prime}$. Hence, we have

$$
\begin{gathered}
u_{t}-\Delta u \geq u(a-u-(\beta+1) \varepsilon), \\
\quad(x, t) \in \Omega \times\left(T_{\varepsilon}^{\prime}, \infty\right), \\
u=0, \quad(x, t) \in \partial \Omega \times\left(T_{\varepsilon}^{\prime}, \infty\right) .
\end{gathered}
$$

Since $\varepsilon$ satisfies (b), we get that

$$
\liminf _{t \rightarrow \infty} u(x, t) \geq u_{(a-(\beta+1) \varepsilon)} .
$$

Thus, from (32), (35), and the arbitrariness of $\varepsilon$, we can conclude that $u(x, t) \rightarrow u_{a}$ as $t \rightarrow \infty$. We finish this proof.

\section{Existence and Nonexistence of Positive Solutions of (2)}

To prove the existence of positive solution of (2) by using fixed point index theory, we need a priori estimate for nonnegative solutions of (1a), (1b), and (1c). So we first give the following theorem.

Theorem 10. Any nonnegative solution $(u(x), v(x), w(x))$ of (2) has a priori bounds:

$$
\begin{gathered}
u(x) \leq a, \\
v(x) \leq c_{4}, \\
w(x) \leq c_{5},
\end{gathered}
$$

where

$$
\begin{aligned}
& c_{4}:=\frac{\beta(a+b) a}{b(1+\beta)}, \\
& c_{5}:=\frac{a(a+d) e \beta}{c d(1+\beta)}+\frac{e c_{4}}{c} .
\end{aligned}
$$

Proof. From the first equation of (2), we have

$$
\begin{aligned}
-\Delta u & \leq u(a-u), \quad x \in \Omega, \\
u & =0, \quad x \in \partial \Omega,
\end{aligned}
$$


and we get $u(x) \leq a$ by maximum principle. Let $v_{1}=\beta u /(1+$ $\beta)+v$ with $\left.v_{1}\right|_{\partial \Omega}=0$. From (lb), we have

$$
\begin{aligned}
-\Delta v_{1} & =\frac{\beta}{1+\beta} u(a-u)-b v-\frac{c w v}{m w+v} \\
& \leq \frac{a \beta u}{1+\beta}-b\left(v_{1}-\frac{\beta u}{1+\beta}\right) \leq \frac{\beta(a+b) a}{1+\beta}-b v_{1} .
\end{aligned}
$$

Thus, by maximum principle, we get

$$
v(x) \leq v_{1}(x) \leq \frac{\beta(a+b) a}{b(1+\beta)}=c_{4} .
$$

We set $w_{1}=(e / c)(\beta u /(1+\beta)+v)+w$ with $\left.w_{1}\right|_{\partial \Omega}=0$, and then we have

$$
\begin{aligned}
-\Delta w_{1} & =\frac{e \beta}{c(1+\beta)} u(a-u)-\frac{b e}{c} v-d w \\
& \leq \frac{a e \beta}{c(1+\beta)} u-d\left[w_{1}-\frac{e \beta u}{c(1+\beta)}-\frac{e v}{c}\right] \\
& \leq \frac{a(a+d) e \beta}{c(1+\beta)}+\frac{d e c_{4}}{c}-d w_{1} .
\end{aligned}
$$

By using maximum principle again, we obtain

$$
w \leq w_{1} \leq \frac{a(a+d) e \beta}{c d(1+\beta)}+\frac{e c_{4}}{c}=c_{5} .
$$

Therefore, we complete this proof.

Now, we introduce some notations:

$$
\begin{aligned}
& E=\left\{C_{0}^{1}(\bar{\Omega})\right\}^{3}, \text { where } C_{0}^{1}(\Omega)=\left\{\phi \in C^{1}(\bar{\Omega}): \phi=0,\right. \\
& \text { on } \partial \Omega\} \text {, } \\
& W=\{K\}^{3} \text {, where } K=\left\{\phi \in C^{1}(\bar{\Omega}): \phi \geq 0\right. \text { in } \\
& \Omega \text { and } \phi=0 \text { on } \partial \Omega\}, \\
& D=\left\{(u, v, w) \in W: u<a+1, v<c_{4}+1, w<c_{5}+1\right\}, \\
& \text { according to Theorem } 10 .
\end{aligned}
$$

Taking

$$
M=\max \left\{a^{2}+a c_{4}+\beta a c_{4}, b c_{4}+\frac{c}{m}, d c_{5}\right\},
$$

we can define a positive and compact operator $F: E \rightarrow E$ by

$$
\begin{aligned}
& F(u, v, w) \\
& =(-\Delta+M)^{-1}\left(\begin{array}{c}
u(a-u-v)-\beta u v+M u \\
\beta u v-b v-\frac{c w v}{m w+v}+M v \\
-d w+\frac{e w v}{m w+v}+M w
\end{array}\right) .
\end{aligned}
$$

It is obvious that $F$ having a positive fixed point in interior of $D$ implies that (2) has a positive solution.

Next, we give the degree of $I-F$ in $D$ relative to $K$ and the fixed point index of $F$ at the trivial solution $(0,0,0)$ of $(1 \mathrm{a})$, (1b), and (1c) relative to $K$.
Lemma 11. Assume that $a>\lambda_{1}$, then one has

(i) $\operatorname{deg}_{W}(1-F, D)=1$,

(ii) $\operatorname{index}_{W}(F,(0,0,0))=0$.

Proof. (i) It is easy to see that $F$ has no fixed point on $\partial \Omega$, so $\operatorname{deg}_{W}(I-F, D)$ is well defined. For $s \in[0,1]$, we define a positive and compact operator $F_{s}: E \rightarrow E$ by

$$
\begin{aligned}
F_{s}(u, v) & (-\Delta+M)^{-1}\left(\begin{array}{c}
s u(a-u-v)-s \beta u v+M u \\
s \beta u v-s b v-\frac{s c w v}{m w+v}+M v \\
-d s w+\frac{s e w v}{m w+v}+M w
\end{array}\right),
\end{aligned}
$$

and then $F_{1}=F$. For each $s$, a fixed point $F_{s}$ is a solution of the following problem:

$$
\begin{aligned}
-\Delta u & =s u(a-u-v)-s \beta u v, \quad x \in \Omega, \\
-\Delta v & =s \beta u v-s b v-\frac{s c w v}{m w+v}, \quad x \in \Omega, \\
-\Delta w & =-d s w+\frac{s e w v}{m w+v}, \quad x \in \Omega, \\
u & =v=0, \quad x \in \partial \Omega .
\end{aligned}
$$

Form Theorem $10 F_{s}$ has no fixed point $\partial \Omega$ and $\operatorname{deg}_{W}(1-$ $\left.F_{s}, D\right)$ is well defined. Hence,

$$
\begin{aligned}
\operatorname{deg}(I-F, D) & =\operatorname{deg}_{W}\left(I-F_{1}, D\right) \\
& =\operatorname{deg}_{W}\left(I-F_{0}, D\right)
\end{aligned}
$$

Note that (46) has only the trivial solution $(0,0,0)$ when $s=0$. Set

$$
L=F_{0}^{\prime}(0,0,0)=(-\Delta+M)^{-1}\left(\begin{array}{ccc}
M & 0 & 0 \\
0 & M & 0 \\
0 & 0 & M
\end{array}\right)
$$

Let $L\left(\xi_{1}, \xi_{2}, \xi_{3}\right)=\left(\xi_{1}, \xi_{2}, \xi_{3}\right)$ for some $\left(\xi_{1}, \xi_{2}, \xi_{3}\right) \in \bar{W}_{(0,0,0)}=$ $K \times K \times K$. Obviously, $\left(\xi_{1}, \xi_{2}, \xi_{3}\right)=(0,0,0)$.

Hence, $I-L$ has no nontrivial kernel in $\bar{W}_{(0,0,0)}$. Since $\lambda_{1}>0$, it is easy to see that $r\left((-\Delta+M)^{-1}(M)\right)<1$ by Proposition 2. It implies that $L$ has no property $\alpha$. Therefore, by Proposition 1, we get the first result of Lemma 11.

(ii) It is obvious that $F(0,0,0)=(0,0,0)$. Let $L=$ $F^{\prime}(0,0,0)$; then

$$
L=(-\Delta+M)^{-1}\left(\begin{array}{ccc}
a+M & 0 & 0 \\
0 & -b+M & 0 \\
0 & 0 & -d+M
\end{array}\right) .
$$


Assume that $L\left(\xi_{1}, \xi_{2}, \xi_{3}\right)=\left(\xi_{1}, \xi_{2}, \xi_{3}\right)$ for some $\left(\xi_{1}, \xi_{2}, \xi_{3}\right) \in$ $\bar{W}_{(0,0,0)}$, and then

$$
\begin{aligned}
-\Delta \xi_{1} & =a \xi_{1}, \quad x \in \Omega, \\
\xi_{1} & =0, \quad x \in \partial \Omega, \\
-\Delta \xi_{2} & =-b \xi_{2}, \quad x \in \Omega, \\
\xi_{2} & =0, \quad x \in \partial \Omega, \\
-\Delta \xi_{3} & =-d \xi_{3}, \quad x \in \Omega, \\
\xi_{3} & =0, \quad x \in \partial \Omega .
\end{aligned}
$$

Since $b>0$ and $d>0$, from (51) and (52), we know that $\xi_{2}=$ $\xi_{3}=0$. If $\xi_{1} \in K$ with $\xi_{1} \neq \equiv 0$, then $a=\lambda_{1}$ from (50), which contradicts with the assumption. Thus $\left(\xi_{1}, \xi_{2}, \xi_{3}\right)=(0,0,0)$. So $I-L$ has nontrivial kernel in $\bar{W}_{(0,0,0)}$.

For $a>\lambda_{1}$, by Proposition 2, we see that $r_{0}=r((-\Delta+$ $\left.M)^{-1}(a+M)\right)>1$, and $r_{0}$ is the principle eigenvalue of $(-\Delta+$ $M)^{-1}(a+M)$ with a corresponding eigenfunction $\phi_{0}>0$. Since $S_{(0,0,0)}=\{(0,0,0)\}$, we see that $\left(\phi_{0},(0,0,0)\right) \in \bar{W}_{(0,0,0)} \backslash$ $S_{(0,0,0)}$. Set $s \in 1 / r_{0} \in(0,1)$, and then $(I-s L)\left(\phi_{0},(0,0,0)\right)=$ $(0,0,0) \in S_{(0,0,0)}$. Thus, $L$ has the property $\alpha$. By Proposition 1 we have index $\operatorname{dex}_{W}(F,(0,0,0))=0$.

Now, we give out the index of the semitrivial solution $\left(u_{a}, 0,0\right)$ of $(2)$.

Lemma 12. Assume that $a>\lambda_{1}$, and then one has

(i) if $-b>\lambda_{1}\left(-\beta u_{a}\right)$, then $\operatorname{index}_{W}\left(F,\left(u_{a}, 0,0\right)\right)=0$;

(ii) if $-b<\lambda_{1}\left(-\beta u_{a}\right)$, then $\operatorname{index}_{W}\left(F,\left(u_{a}, 0,0\right)\right)=1$.

Proof. Let $L=F^{\prime}\left(u_{a}, 0,0\right)$; then

$$
\begin{aligned}
L= & (-\Delta+M)^{-1} \\
& \quad\left(\begin{array}{ccc}
a-2 u_{a}+M & -(1+\beta) u_{a} & 0 \\
0 & \beta u_{a}-b+M & 0 \\
0 & 0 & -d+M
\end{array}\right) .
\end{aligned}
$$

Let $L\left(\xi_{1}, \xi_{2}, \xi_{3}\right)=\left(\xi_{1}, \xi_{2}, \xi_{3}\right)$ for some $\left(\xi_{1}, \xi_{2}, \xi_{3}\right) \in \bar{W}_{\left(u_{a}, 0,0\right)}=$ $C_{0}^{1}(\bar{\Omega}) \times K \times K$. Then

$$
\begin{aligned}
-\Delta \xi_{1}+\left(2 u_{a}-a\right) \xi_{1} & =-(1+\beta) u_{a} \xi_{2}, \quad x \in \Omega, \\
-\Delta \xi_{2}+\left(b-\beta u_{a}\right) \xi_{2} & =0, \quad x \in \Omega, \\
-\Delta \xi_{3}+d \xi_{3} & =0, \quad x \in \Omega, \\
\xi_{1} & =\xi_{2}=\xi_{3}=0, \quad x \in \partial \Omega .
\end{aligned}
$$

Since $\xi_{2}, \xi_{3} \in K,-b \neq \lambda_{1}\left(-\beta u_{a}\right)$ and $d>0$. So we get that $\xi_{2}=\xi_{3}=0$. Hence, from the first equation of (54) we have

$$
\begin{gathered}
-\Delta \xi_{1}+\left(2 u_{a}-a\right) \xi_{1}=-(1+\beta) u_{a} \xi_{2}, \quad x \in \Omega \\
\xi_{1}=0, \quad x \in \partial \Omega .
\end{gathered}
$$

If $\xi_{1} \neq 0$, then $\lambda_{1}\left(2 u_{a}-a\right)=0$ by Proposition 2 . On the other hand, $\lambda_{1}\left(2 u_{a}-a\right)>\lambda_{1}\left(u_{a}-a\right)=0$, and we get a contradiction. Hence, $\left(\xi_{1}, \xi_{2}, \xi_{3}\right)=(0,0,0)$. Then $I-L$ has no nontrivial kernel in $\bar{W}_{\left(u_{a}, 0,0\right)}$.

(i) Since $-b>\lambda_{1}\left(-\beta u_{a}\right)$, by Proposition 1 , we have $r_{0}=$ $r\left((-\Delta+M)^{-1}\left(\beta u_{a}-b+M\right)\right)>1$ which is an eigenvalue of $(-\Delta+M)^{-1}\left(\beta u_{a}-b+M\right)$ with a corresponding eigenfunction $\phi_{0}>0$. Since $S_{\left(u_{a}, 0,0\right)}=C_{0}^{1}(\bar{\Omega}) \times\{0\} \times\{0\}$, we have $\left(0, \phi_{0}, 0\right) \in$ $\bar{W}_{\left(u_{a}, 0,0\right)} \backslash S_{\left(u_{a}, 0,0\right)}$. Set $s \in 1 / r_{0} \in(0,1)$, and then

$$
\begin{aligned}
(I-s L) & \left(\begin{array}{c}
0 \\
\phi_{0} \\
0
\end{array}\right) \\
& =\left(\begin{array}{c}
0 \\
\phi_{0} \\
0
\end{array}\right)-s(-\Delta+M)^{-1}\left(\begin{array}{c}
-(1+\beta) u_{a} \phi_{0} \\
\left(\beta u_{a}-b+M\right) \phi_{0} \\
0
\end{array}\right) \\
& =\left(\begin{array}{c}
s(-\Delta+M)^{-1}(1+\beta) u_{a} \phi_{0} \\
0 \\
0
\end{array}\right) \in S_{\left(u_{a}, 0,0\right)} .
\end{aligned}
$$

Hence, $L$ has the property $\alpha$ on $\bar{W}_{\left(u_{a}, 0,0\right)}$ resulting in $\operatorname{index}_{W}\left(F,\left(u_{a}, 0,0\right)\right)=0$ according to Proposition 1 .

(ii) First, we prove that $L$ does not have the property $\alpha$ on $\bar{W}_{\left(u_{a}, 0,0\right)}$.

Since $-b<\lambda_{1}\left(-\beta u_{a}\right)$, we have $r\left((-\Delta+M)^{-1}\left(-\beta u_{a}\right)\right)<$ 1. On the contrary, we suppose that $L$ has the property $\alpha$ on $\bar{W}_{\left(u_{a}, 0,0\right)}$. Then there exist $s \in(0,1)$ and $\left(\xi_{1}, \xi_{2}, \xi_{3}\right) \in \bar{W}_{\left(u_{a}, 0,0\right)}$ such that $(I-s L)\left(\xi_{1}, \xi_{2}, \xi_{3}\right) \in S_{\left(u_{a}, 0,0\right)}$. So

$$
(-\Delta+M)^{-1}\left(\beta u_{a}-b+M\right) \xi_{2}=\frac{\xi_{2}}{s} .
$$

Then $1 / s$ is an eigenvalue of the operator $(-\Delta+M)^{-1}\left(\beta u_{a}-\right.$ $b+M)$, which contradicts $r\left((-\Delta+M)^{-1}\left(\beta u_{a}-b+M\right)\right)<1$. So $L$ does not have the property $\alpha$ on $\bar{W}_{\left(u_{a}, 0,0\right)}$. By Proposition 2 , we have $\operatorname{index}_{W}\left(F,\left(u_{a}, 0,0\right)\right)=(-1)^{\sigma}$, where $\sigma$ is the sum of the multiplicities of all eigenvalues of $L$. Next, we will prove that $\sigma=0$.

Assume that $1 / \rho>1$ is an eigenvalue of $L$ with corresponding eigenfunction $\left(\xi_{1}, \xi_{2}, \xi_{3}\right)$, and then $L\left(\xi_{1}, \xi_{2}, \xi_{3}\right)=$ $1 / \rho\left(\xi_{1}, \xi_{2}, \xi_{3}\right)$; that is,

$$
\begin{aligned}
-\Delta \xi_{1}+M \xi_{1} & =\rho\left(\left(a-2 u_{a}+M\right) \xi_{1}-(1+\beta) u_{a} \xi_{2}\right), \\
x \in \Omega, & \\
-\Delta \xi_{2}+M \xi_{2} & =\rho\left(\beta u_{a}-b+M\right) \xi_{2}, \quad x \in \Omega, \\
-\Delta \xi_{3}+M \xi_{3} & =\rho(-d+M) \xi_{3}, \quad x \in \Omega, \\
\xi_{1} & =\xi_{2}=\xi_{3}=0, \quad x \in \partial \Omega .
\end{aligned}
$$

Since $0<\rho<1$ and $d>0$, it follows from the third equation (58) that $\xi_{3}=0$. 
We suppose that $\xi_{2} \neq 0$, and it follows from the second equation of (58) and Proposition 2 that

$$
0=\lambda_{1}\left(M(1-\rho)-\rho\left(\beta u_{a}-b\right)\right)>\lambda_{1}\left(-\beta u_{a}\right)+b,
$$

which contradicts $-b<\lambda_{1}\left(-\beta u_{a}\right)$, and so $\xi_{2}=0$.

If $\xi_{1} \neq 0$, it follows from the first equation of (58) that

$$
\begin{aligned}
0 & =\lambda_{1}\left(M(1-\rho)-\rho\left(a-2 u_{a}\right)\right)>\lambda_{1}\left(2 u_{a}-a\right) \\
& >\lambda_{1}\left(u_{a}-a\right)=0 .
\end{aligned}
$$

Thus, we get the contradiction and this implies that $\xi_{1}=$ 0 . Hence, $\left(\xi_{1}, \xi_{2}, \xi_{3}\right)=(0,0,0)$, which implies that $L$ has no eigenvalue being greater than 1 ; that is, $\sigma=0$, and index $_{W}\left(F,\left(u_{a}, 0,0\right)\right)=1$. We complete the proof.

Next, we consider the other semitrivial solutions of (2). Let us consider the following three subsystems:

$$
\begin{aligned}
-\Delta u & =u(a-u-v)-\beta u v, \quad x \in \Omega, \\
-\Delta v & =\beta u v-b v, \quad x \in \Omega, \\
u & =v=0, \quad x \in \partial \Omega . \\
-\Delta u & =u(a-u), \quad x \in \Omega, \\
-\Delta w & =-d w, \quad x \in \Omega, \\
u & =w=0, \quad x \in \partial \Omega . \\
-\Delta v & =-b v-\frac{c w v}{m w+v}, \quad x \in \Omega, \\
-\Delta w & =-d w+\frac{e w v}{m w+v}, \quad x \in \Omega, \\
v & =w=0, \quad x \in \partial \Omega .
\end{aligned}
$$

It is obvious that $(63)$ has trivial solution $(v, w)=(0,0)$ and that (62) only has a trivial solution $(u, w)=(0,0)$ and a semitrivial solution $(u, w)=\left(u_{a}, 0\right)$ if and only if $a>\lambda_{1}$. For subsystem (61), a lot of papers $[15,22]$ and references wherein have discussed systems in this case, so we directly give out some results about (61).

Theorem 13. Equation (61) has a positive solution $\left(u^{*}, v^{*}\right)$ with $u^{*}<u_{a}$ if and only if $a>\lambda_{1}$ and $-b>\lambda_{1}\left(-\beta u_{a}\right)$. Moreover, if $a-\beta-1>\lambda_{1}$ and $-b>\lambda_{1}\left(-\beta u_{(a-\beta-1)}\right)$, then the positive solution $\left(u^{*}, v^{*}\right)$ satisfies $u_{(a-\beta-1)} \leq u^{*}$ and $\bar{v} \leq v^{*}$, where $\bar{v}$ is the unique positive solution of the following equations:

$$
\begin{aligned}
-\Delta v & =v\left(\beta u_{(a-\beta-1)}-b\right), \quad x \in \Omega, \\
v & =0, \quad x \in \partial \Omega .
\end{aligned}
$$

Let $\{(u, v)\}$ be the set of all positive solutions of (61), denoted by $\Phi$, and $\Psi=\{(u, v, 0),(u, v) \in \Phi\}$. It is easy to see that (61) has semitrivial solution $\left(u^{*}, v^{*}, 0\right) \in \Psi$ from Theorem 13. Next, we give the degree of $(I-F)$ in $\Psi$ relative to $W$.
Lemma 14. Assume $\Psi \neq \emptyset$, and then one has the following:

(i) if $-d>\lambda_{1}-e$, for any $\left(u^{*}, v^{*}, 0\right) \in \Psi$, then $\operatorname{deg}_{W}(I-$ $F, \Psi)=0$;

(ii) if $-d<\lambda_{1}-e$, for any $\left(u^{*}, v^{*}, 0\right) \in \Psi$, then $\operatorname{deg}_{W}(I-$ $F, \Psi)=1$.

Proof. Let

$$
\begin{aligned}
E_{1} & =\left\{C_{0}^{1}(\bar{\Omega})\right\}^{2}, \\
E_{2} & =C_{0}^{1}(\bar{\Omega}), \\
W_{1} & =\{K\}^{2}, \\
W_{2} & =K .
\end{aligned}
$$

Define

$$
\begin{aligned}
& F_{1}(u, v, w) \\
& =(-\Delta+M)^{-1}\left(\begin{array}{c}
u(a-u)-\beta u v+M u \\
\beta u v-b v-\frac{c v w}{m w+v}+M v
\end{array}\right), \\
& F_{2}(u, v, w)=(-\Delta+M)^{-1}\left(\frac{e v w}{m w+v}-d w\right) .
\end{aligned}
$$

Then $F=\left(F_{1}, F_{2}\right)$ and we are in the framework to use Theorem 3. We choose a neighborhood $U \subset W_{1} \cap D$ of $\Psi \cap W_{1}$ such that $\left(u_{a}, 0\right) \notin \bar{U}$.

Now, $F_{1}(u, v, 0)=(u, v)$ with $(u, v) \in \bar{U}$ if and only if $(u, v, 0) \in \Psi$. By the results in [22], we have

$$
\begin{aligned}
& \operatorname{deg}_{W_{1}}\left(I-\left.F_{1}\right|_{W_{1}}, U, 0\right) \\
& \quad= \begin{cases}1, & \text { if } a>\lambda_{1},-b>\lambda_{1}\left(-\beta u_{a}\right), \\
-1, & \text { if } a<\lambda_{1},-b<\lambda_{1}\left(-\beta u_{a}\right), \\
0, & \text { if }\left(a-\lambda_{1}\right) \cdot\left[-b-\lambda_{1}\left(-\beta u_{a}\right)\right]<0 .\end{cases}
\end{aligned}
$$

For any $\left(u^{*}, v^{*}, 0\right) \in \Psi$, a direct calculation shows that

$$
\left.F_{2}^{\prime}\left(u^{*}, v^{*}, 0\right)\right|_{W_{2}} w=(-\Delta+M)^{-1}(M-b+e) w .
$$

Notice that, by our choice of $M, M-b+e>0$, so it follows from the maximum principle that $\left.F_{2}^{\prime}\left(u^{*}, v^{*}, 0\right)\right|_{W_{2}}$ is $u_{0}$-positive in the sense of [23] with $u_{0}=(-\Delta)^{-1}$. Hence, $r\left(\left.F_{2}^{\prime}\left(u^{*}, v^{*}, 0\right)\right|_{W_{2}}\right)>0$ and is the only eigenvalue corresponding to a positive eigenvector. By a simple calculation, we can easily show that $r\left(\left.F_{2}^{\prime}\left(u^{*}, v^{*}, 0\right)\right|_{W_{2}}\right)>1$ if and only if $-d>$ $\lambda_{1}-e$ and $r\left(\left.F_{2}^{\prime}\left(u^{*}, v^{*}, 0\right)\right|_{W_{2}}\right)<1$ if and only if $-d<\lambda_{1}-e$. Therefore, by Theorem 3 and the discussion above, we have

$$
\begin{aligned}
& \operatorname{deg}_{W}\left(I-F, U \times W_{2}(\varepsilon), 0\right) \\
&= \begin{cases}0, & \text { if }-d>\lambda_{1}-e, \\
\operatorname{deg}_{W}\left(I-\left.F_{1}\right|_{W_{1}}, U, 0\right), & \text { if }-d<\lambda_{1}-e .\end{cases}
\end{aligned}
$$

Again since the degree does not depend on the particular choice of $U$ and $\varepsilon, \Psi \neq \emptyset$ means $a>\lambda_{1}$ and $-b>\lambda_{1}\left(-\beta u_{a}\right)$. Thus, we complete this proof. 
From the discussion above, we can obtain the existence of positive solutions of (2).

Theorem 15. Assume $a>\lambda_{1}$. Equation (2) has at least one positive solution if

$$
\begin{aligned}
a & >\lambda_{1}+\beta+1, \\
-b & >\lambda_{1}\left(-\beta u_{(a-\beta-1)}\right), \\
-d & >\lambda_{1}-e
\end{aligned}
$$

and then (2) has at least one positive solution.

Proof. Since $a>\lambda_{1}$, we obtain $\operatorname{deg}_{W}(I-F, D)=1$ and index $_{W}(F,(0,0,0))=0$ from Lemma 11. Hence, we have

$$
\operatorname{index}_{W}\left(F,\left(u_{a}, 0,0\right)\right)+\operatorname{deg}_{W}(I-F, D)=1 .
$$

Since $-b>\lambda_{1}\left(-\beta u_{(a-\beta-1)}\right)>\lambda_{1}\left(-\beta u_{a}\right)$, we have $\operatorname{index}_{W}(F$, $\left.\left(u_{a}, 0,0\right)\right)=0$ from Lemma 12. Moreover, Theorem 13 implies $\Psi \neq \emptyset$. Let $\left(u^{*}, v^{*}, 0\right) \in \Psi$. Since $u^{*} \geq u_{(a-\beta)}$ and $v^{*} \geq \bar{v}$ by Theorem 13, we have $-d>\lambda_{1}-e$ and thus $\operatorname{deg}_{W}(I-F, \Psi)=0$ from Lemma 14. Therefore, $\operatorname{index}_{W}\left(F,\left(u_{a}, 0,0\right)\right)+\operatorname{deg}_{W}(I-$ $F, \Psi)=0$. So (71) holds. We complete this proof.

Next, we give out the result of nonexistence of positive solutions.

Theorem 16. If any one of the following conditions holds, then (2) has no positive solution:

(i) $a<\lambda_{1}$;

(ii) $a>\lambda_{1}$ and $-b \leq \lambda_{1}\left(-\beta u_{a}\right)$.

Proof. Assume that (2) has positive solution $(u, v, w)$, and then $u, v, w$ satisfy the following three equations, respectively:

$$
\begin{aligned}
-\Delta u & =u(a-u-v)-\beta u v, \quad x \in \Omega, \\
u & =0, \quad x \in \partial \Omega, \\
-\Delta v & =\beta u v-b v-\frac{c w v}{m w+v}, \quad x \in \Omega, \\
v & =0, \quad x \in \partial \Omega, \\
-\Delta w & =\frac{e w v}{m w+v}-d w, \quad x \in \Omega, \\
w & =0, \quad x \in \partial \Omega .
\end{aligned}
$$

(i) Since $u>0$ in $\Omega$, we get from (72) that

$$
a=\lambda_{1}(u+(\beta+1) v)>\lambda_{1} .
$$

By Proposition 2 and comparison property of principle eigenvalue, this contradicts $a<\lambda_{1}$. Then (73) and (74) have $v=0$ and $w=0$.

(ii) Since $v>0$ in $\Omega$, we get from (73) that

$$
-b=\lambda_{1}\left(\frac{c w}{m w+v}-\beta u\right)>\lambda_{1}\left(-\beta u_{a}\right),
$$

by Proposition 2 and comparison property of principle eigenvalue, we get a contradiction $-b \leq \lambda_{1}\left(-\beta u_{a}\right)$.

\section{Competing Interests}

The authors declare that they have no competing interests.

\section{Acknowledgments}

Thanks are due to reviewers' comments and suggestions for improving this paper. This work is supported by Grants 61563033,11563005 , and 71363043 from the National Natural Science Foundation of China and 20161BAB201010, 20151BAB212011, and 20151BAB201021 from the Natural Science Foundation of Jiangxi Province.

\section{References}

[1] J. D. Murray, Mathematical Biology. I. An Introduction, vol. 17 of Interdisciplinary Applied Mathematics, Springer, New York, NY, USA, 2002.

[2] Y. Li and D. Xiao, "Bifurcations of a predator-prey system of Holling and Leslie types," Chaos, Solitons \& Fractals, vol. 34, no. 2, pp. 606-620, 2007.

[3] M. Liao, X. Tang, and C. Xu, "Stability and instability analysis for a ratio-dependent predator-prey system with diffusion effect," Nonlinear Analysis: Real World Applications, vol. 12, no. 3, pp. 1616-1626, 2011.

[4] R. M. Anderson and R. M. May, Infectious Diseases of Humans, Volume 1, Oxford University Press, Oxford, UK, 1991.

[5] L. Rass and J. Radcliffe, Spatial Deterministic Epidemics, vol. 102, American Mathematical Society, 2003.

[6] K. P. Hadeler and H. I. Freedman, "Predator-prey populations with parasitic infection," Journal of Mathematical Biology, vol. 27, no. 6, pp. 609-631, 1989.

[7] Y. Xiao and L. Chen, "Modeling and analysis of a predator-prey model with disease in the prey," Mathematical Biosciences, vol. 171, no. 1, pp. 59-82, 2001.

[8] Y. Xiao and L. Chen, "A ratio-dependent predator-prey model with disease in the prey," Applied Mathematics and Computation, vol. 131, no. 2-3, pp. 397-414, 2002.

[9] H. Yin, X. Xiao, and X. Wen, "Turing patterns in a predatorprey system with self-diffusion," Abstract and Applied Analysis, vol. 2013, Article ID 891738, 10 pages, 2013.

[10] H. Yin, J. Zhou, X. Xiao, and X. Wen, "Analysis of a diffusive Leslie-Gower predator-prey model with nonmonotonic functional response," Chaos, Solitons \& Fractals, vol. 65, pp. 51-61, 2014.

[11] H. Yin, X. Xiao, X. Wen, and K. Liu, "Pattern analysis of a modified Leslie-Gower predator-prey model with Crowley-Martin functional response and diffusion," Computers \& Mathematics with Applications, vol. 67, no. 8, pp. 1607-1621, 2014.

[12] X. Zhang, Y. Huang, and P. Weng, "Permanence and stability of a diffusive predator-prey model with disease in the prey," Computers and Mathematics with Applications, vol. 68, no. 10, pp. 1431-1445, 2014.

[13] J. Zhou and J. Shi, "The existence, bifurcation and stability of positive stationary solutions of a diffusive LeslieGower predator-prey model with Holling-type II functional responses," Journal of Mathematical Analysis and Applications, vol. 405, no. 2, pp. 618-630, 2013.

[14] J. Zhou, "Positive solutions of a diffusive predator-prey model with modified Leslie-Gower and Holling-type II schemes," 
Journal of Mathematical Analysis and Applications, vol. 389, no. 2, pp. 1380-1393, 2012.

[15] J. Zhou and C. Mu, "Positive solutions for a three-trophic food chain model with diffusion and Beddington-Deangelis functional response," Nonlinear Analysis: Real World Applications, vol. 12, no. 2, pp. 902-917, 2011.

[16] L. Li, "Coexistence theorems of steady states for predator-prey interacting systems," Transactions of the American Mathematical Society, vol. 305, no. 1, pp. 143-166, 1988.

[17] E. N. Dancer, "On the indices of fixed points of mappings in cones and applications," Journal of Mathematical Analysis and Applications, vol. 91, no. 1, pp. 131-151, 1983.

[18] M. Wang and Q. Wu, "Positive solutions of a prey-predator model with predator saturation and competition," Journal of Mathematical Analysis and Applications, vol. 345, no. 2, pp. 708718, 2008.

[19] E. N. Dancer and Y. Du, "Positive solutions for a threespecies competition system with diffusion-I. General existence results," Nonlinear Analysis: Theory, Methods \& Applications, vol. 24, no. 3, pp. 337-357, 1995.

[20] A. Pazy, Semigroups of Linear Operators and Applications to Partial Differential Equations, vol. 44 of Applied Mathematical Sciences, Springer, New York, NY, USA, 1983.

[21] J. W. Cholewa and T. Dlotko, Global Attractors in Abstract Parabolic Problems, vol. 278, Cambridge University Press, 2000.

[22] E. N. Dancer, "On positive solutions of some pairs of differential equations," Transactions of the American Mathematical Society, vol. 284, no. 2, pp. 729-743, 1984.

[23] M. A. Krasnoselskij, Positive Solutions of Operator Equations, Noordhoff, Groningen, The Netherlands, 1964. 


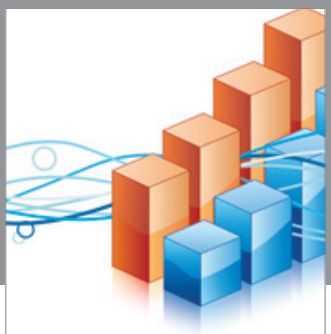

Advances in

Operations Research

vatem alat4

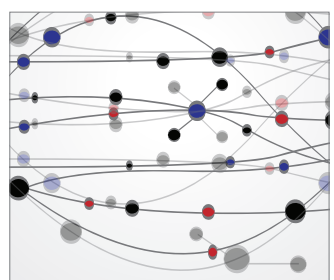

\section{The Scientific} World Journal
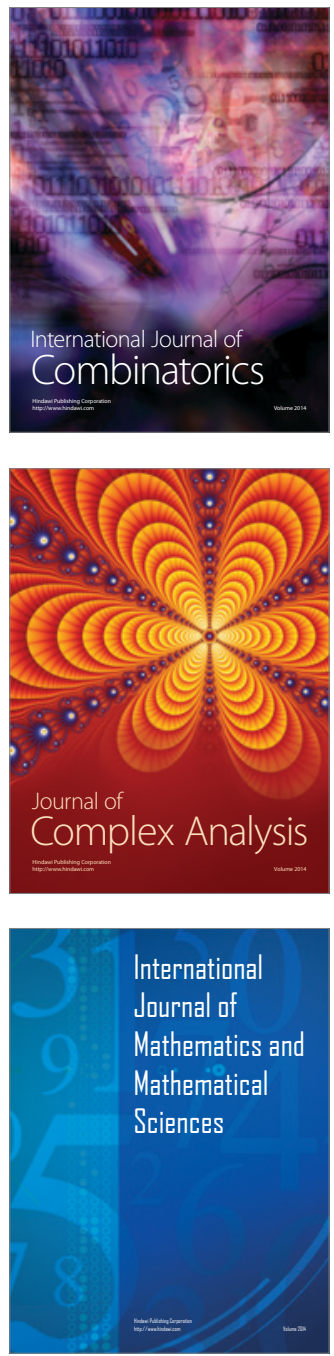
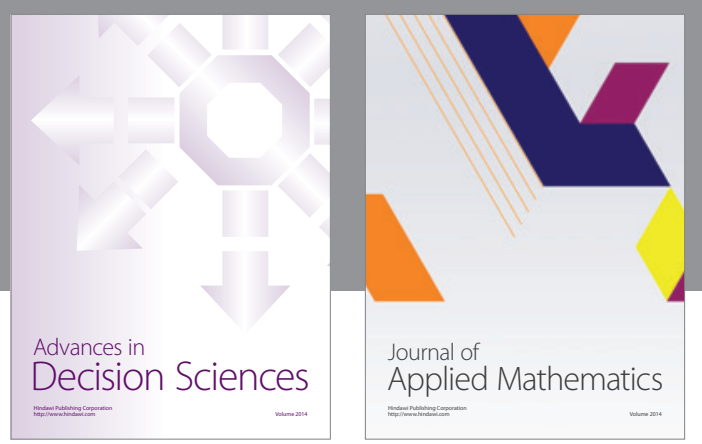

Algebra

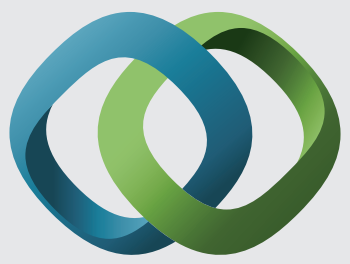

\section{Hindawi}

Submit your manuscripts at

http://www.hindawi.com
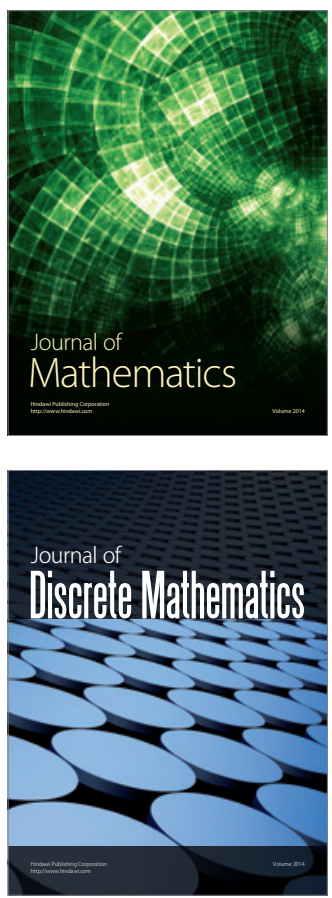

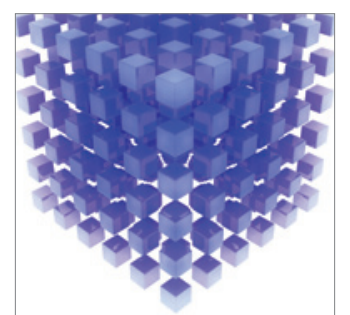

Mathematical Problems in Engineering
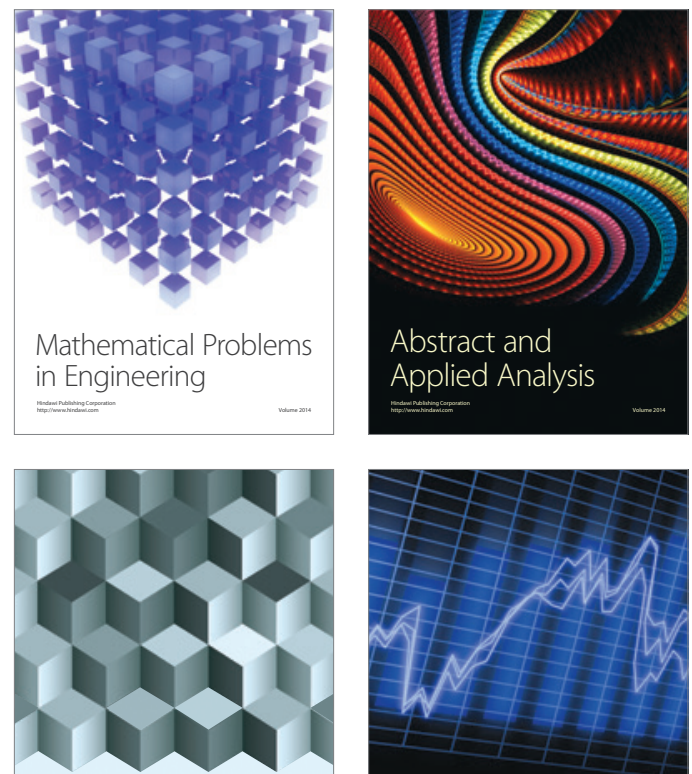

Journal of

Function Spaces

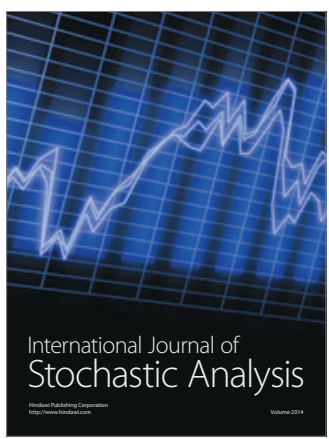

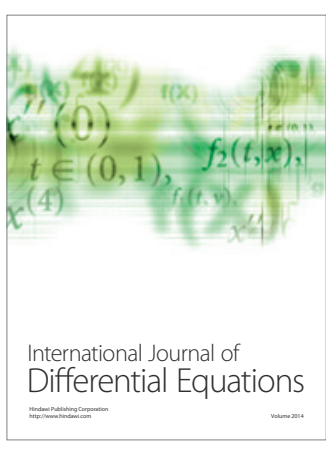
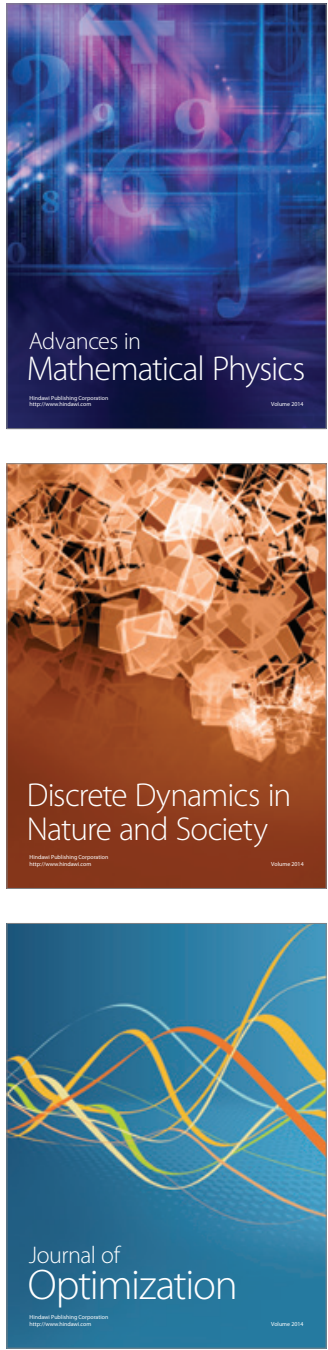